\title{
A Context-Aware Framework for Patient Navigation and Engagement (CANE)
}

\author{
Wen Yao, Jerome Rolia, Sujoy Basu, Sharad Singhal \\ Services and Solutions Lab, HP Labs \\ Palo Alto, CA 94304, USA \\ \{wen.yao2, jerry.rolia, sujoy.basu, \\ sharad.singhal\}@hp.com
}

\author{
Akhil Kumar \\ Smeal College of Business, Penn State University \\ University Park, PA 16802, USA \\ akhilkumar@psu.edu
}

\begin{abstract}
Engaging patients in the management of their health care can improve the quality of their care and enhance their experience while making more efficient use of care provider resources, especially for chronic diseases. However, health care system complexity and the challenge of consumer health literacy hinder greater engagement. In this paper, we propose a ContextAware patient Navigation and Engagement (CANE) framework to support informed decision making and more efficient use of health care services. We use the BPMN 2.0 workflow language to formalize the description of navigation processes derived from medical guidelines and develop a healthcare network ontology to assist patient understanding of various clinical activities from multiple perspectives (e.g., resource, logistics, financial, and time). A running example of type 2 diabetes management is used to illustrate our CANE framework. Finally, we describe the system architecture of our prototype.
\end{abstract}

Keywords-patient navigation; context-aware; decision support; clinical process; ontology

\section{INTRODUCTION}

Despite advances in life expectancy and quality of life, the current healthcare delivery system faces significant challenges in terms of cost, accessibility and quality [1]. A study [2] showed that healthcare expenditures in 2009 exceeded $17 \%$ of GDP in the U.S. and the figure is estimated to reach $25 \%$ by 2025. In view of a rapidly aging population and greater incidence of chronic illnesses, many have proposed consumercentric care delivery to reduce the burden on the health care system. There is an emerging consensus in the health policy community that engaged and informed consumers have a vital role to play in improving the quality of care [3]. Further, rapidly growing patient interest in having access to their health data and participating in their care process, a.k.a. shared decision making, has motivated health organizations to provide patient-oriented care delivery both in clinical and homecare settings. Unfortunately, as the health care system becomes more complex (e.g., involving multiple providers, insurance companies, and their interactions), it also makes consumer engagement even harder. Moreover, medical guidelines and terms used by clinicians are difficult for the ordinary patient to understand. This gives rise to a need for a system that supports patient navigation, education and participation.

Patient navigation is defined as "the process(es) by which patients and/or their caregivers move into and through multiple parts of the health care enterprise in order to gain access to and use its services in a manner that maximizes the likelihood of gaining the positive health outcomes available through those services." [4] It refers to the assistance offered to patients as they receive services from a complex healthcare system to overcome barriers in accessing quality care and treatment, e.g. navigating the medical guidelines associated with their conditions, along with possible treatment options and their providers, insurance coverage and out of pocket costs, financial support arrangements, etc. Such navigation has usually been guided by a human agent, often in cancer management to reduce health disparities [5], and not by an automated agent as we propose in this study. Multiple stakeholders participate in this enterprise system, including patients (or broadly, consumers), providers, insurance companies, and policy makers. Currently, most patient-oriented systems are primarily Personal Health Record (PHR) systems [6, 7] which allow patients to track their health information and to communicate with their care providers. The PHR is initially populated by downloading information from Electronic Health Record (EHR) systems maintained by healthcare organizations. PHR systems help patients to store, view and share their medical histories, medications, lab results, etc., but they provide hardly any decision support functionality to help them make decisions through a better understanding of their medical situation.

In recent years, health professionals have been increasingly advised, if not required, to follow standard medical guidelines in their practice. In academia, there is a growing interest within the health informatics research community in developing computer interpretable guidelines (CIGs) and decision support systems that use CIGs, reviewed in [8,9]. Most of these systems are designed for health professionals rather than for patients. CIGs are not easy for patients to comprehend. The NICE pathways [10], developed by National Institute for Health and Clinical Excellence in UK, display medical guidelines as flow charts. They offer an interactive tool that provides fast access to medical guidance for health and social care professionals. Patients may use this tool to learn more details about their current health problems and the alternative choices they have. However, patients can easily get lost or confused since the tool is not aware of the actual patient context. These systems can lead to more informed choices about treatment alternatives but are not able to manage health issues in a systematic manner because every patient's situation is unique [11] and every healthcare system is unique. Thus, there is an urgent need to develop an integrated system to help 
patients navigate the intricacies of their healthcare system in a context-aware manner.

With the goal of giving consumers a more proactive role in their own health care, this paper proposes a Context-Aware Navigation and Engagement (CANE) framework for patients to manage their health care in a systematic manner. We focus mainly on navigation issues pertaining to the understanding of medical guidelines as they relate to the patient's unique situation or context. Context-aware navigation means assisting patients with self-management guided by appropriate medical knowledge based on their context, including medical history, current conditions, personal preferences, etc. Self-management refers to self-monitoring and decision making by patients, which can lead to improvements in health outcomes and reductions in healthcare costs, especially for chronic diseases. To fulfill this vision, we formalize patient navigation processes adapted from medical guidelines in a formal workflow language called BPMN 2.0 [12], and develop a healthcare network ontology to assist patients' understanding of various clinical activities performed by care providers in a healthcare system from different perspectives, e.g., resource, logistics, financial, time, etc. Throughout this paper, we use a running example of managing type 2 diabetes to illustrate our approach, and show how this method can help patients better manage their health matters and receive care services.

The organization of this paper is as follows. Section II gives a running example that illustrates what we mean by contextaware navigation. Our CANE approach is presented in Section III, including a conceptual framework, a patient context model, a meta-model for navigation activities and a healthcare network ontology. Next, Section IV shows how context-aware navigation is realized using the CANE framework. In Section $\mathrm{V}$, we describe the system architecture for prototype implementation. A review of related work follows in Section VI. Finally, summary and concluding remarks are offered in Section VII.

\section{PRELIMINARIES}

\section{A. The Need for Context-aware Navigation}

We identify and analyze the patient need for context-aware navigation by analyzing the literature concerning patient care. According to the review of literature in [13], "patient-centered interaction promote adherence and lead to improved health outcomes". They further concluded that the individualization of patient care and patient involvement are key characteristics in patient-centered care. However, the complexity of the health care system and lack of consumer health literacy [14] are the major barriers to achieving this goal. Moreover, with each patient's unique situation [11], and with patient's daily routine change [15], the patient pathway might be affected (e.g., different treatment methods due to allergies, or change of treatment during a busy business trip). Thus, identifying various patient contexts and using them to support patient navigation through the health care system in a context-aware manner are essential to enhance patient experience and improve healthcare outcomes.

The concept of context-awareness has been extensively studied in different application domains, including smart space, information systems, mobile commerce, communication systems, etc. [16]. The most popular and widely used contextaware application is in a pervasive computing environment or smart space, including smart home, smart hospital, campus, museum, and conference room where sensors are installed to collect contextual data and send it to the server via wireless network. Once the context changes (e.g., a doctor walks into the surgery room), these context-aware applications can adapt their behavior by different actions (e.g., show the patient record on the computer screen in the operating room). A comprehensive survey of context-aware applications can be found in Baldauf et al. [17] and Hong et al. [16]'s work. In particular, the idea of context-awareness has been applied in hospitals, such as a context-aware pill container and a contextaware hospital bed [18]. Other examples were also discussed.

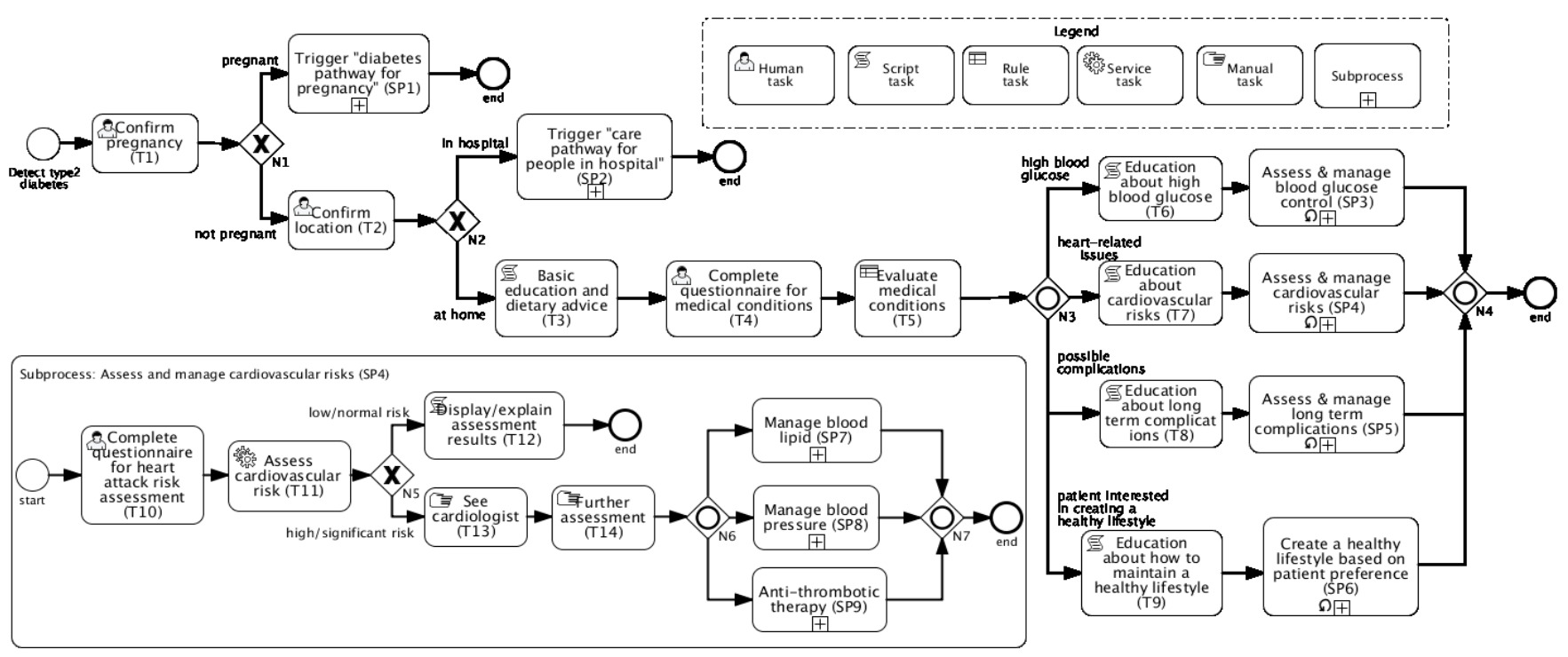

Figure 1. A navigation process for managing type 2 diabetes patients using BPMN 2.0 notations 
Context-aware applications benefit consumers by delivering personalized information as per individual needs. To the best of our knowledge, we haven't found any studies that propose to apply context-awareness in patient navigation, despite the urgent patient needs in this domain as discussed previously.

\section{B. A Running Example}

This section introduces our running example of managing type 2 diabetes. We chose this case because it develops slowly and is a very common disease, especially in inactive people with excess body weight. Moreover, type 2 diabetes can lead to severe complications. It is reported in [19] that "three out of every 5 patients with type 2 diabetes suffer from at least one significant complication of the disease, such as heart disease, stroke, eye damage, chronic kidney disease or foot problems leading to amputation." We use this example to illustrate the aspects of patient navigation we focus on.

Figure 1 shows a navigation process that corresponds to medical guidelines for treating patients with type 2 diabetes. It shows how a patient with type 2 diabetes goes through different steps and procedures based on their personal context, from the onset of the disease until the patient becomes stable or is cured. First, the patient confirms whether she is pregnant (T1). If so, she is directed to the "diabetes pathway for pregnancy" subprocess (SP1) for which the details are omitted here; else, she will progress based on her current care location which is determined next (T2). A patient in a hospital is led along another navigation guideline, "care pathway for people in hospital (SP2)." For home care, the patient receives basic education and dietary advice (T3), and then completes a questionnaire for assessing her medical condition (T4). Next, rules that encode this medical knowledge are triggered and generate the evaluation results (T5), based on which one or more subsequent paths are executed. For example, if the patient has high blood glucose, related education will be given (T6), followed by further assessment and management of blood glucose (SP3). Similarly, other patient context information may trigger additional navigation activities, e.g. heart-related problems will lead to cardiovascular risks assessment and management. This is the basic idea behind context-aware navigation.

The navigation process described above is captured by a BPMN 2.0 [12] workflow model that is comprised of activities and nodes. BPMN 2.0 is a de facto standard now for process modeling and it can support various types of tasks and workflow patterns. It also provides a flowchart-like graphical representation. Thus, we use BPMN 2.0 notations to model the navigation processes. Different types of navigation activities are modeled by various types of task, such as human task, service task, rule task, etc. (shown in the legend in Figure 1), while routing nodes can be formalized using various gateway notations. For example, human tasks, such as T1, T2 and T4, allow a patient to interact with the system by either confirming that the data in the context database is correct, or entering new data readings for, say, blood pressure, temperature, etc. A rule task (such as T5 to evaluate a medical condition) is associated with a number of rules that encode medical knowledge for rulebased reasoning to support decision making. A script task (e.g., $\mathrm{T} 3$ to show relevant links for education and dietary advice) could be a code snippet in JavaScript or another language to realize a certain function, while a service task reaches the same goal through an implemented Web service, e.g., T11 for assessing cardiovascular risks as realized by a service offered by American Heart Association. At a certain stage, the patient may need to see a provider and receive professional care services. For example, T13 is a manual task meaning the patient needs to see a cardiologist in person if her cardivascular risk is high or significant. As a result, a patient may visit multiple providers for different purposes. Invocation of another navigation process is enabled by subprocesses. For example, the subprocess SP4 (assess and manage cardiovascular risks) is expanded, as shown in the lower left box in Figure 1. More details of BPMN 2.0 notations can be found in [12].

During the navigation process, the system keeps collecting and updating various contextual data obtained through patient conversation or from various providers that deliver care services. It presents customized educational materials and instructions for receiving care services. For instance, if the guideline mentions a certain lab test, a patient may wish to know the medical reason for this test, where it will be performed and by whom, how long it takes, the cost of the test and whether it is covered by insurance, etc. By making it easy to get such information our approach allows patients to have a unified and integrated view of their current issue and ensures their treatment follows best practice guidelines (i.e., evidencebased).

\section{THE CANE APPROACH}

Figure 2 shows the conceptual model that serves as a roadmap for the CANE approach. It describes the key concepts in our framwork and the semantic relationships among them.

A navigation process is composed of navigation activities such as: patient inquiry, decision and actions. We use the patient inquiry activity to collect various aspects of the patient context (e.g., body weight, blood pressure, history of heart attacks), including process context (i.e., the current step/stage in the navigation process), personal context (i.e., their health information and preferences), logistics context (i.e., their current location), and social context (i.e., information about their family and friends). The patient context is stored in the PHR database that is augmented with a preference data schema. During navigation, the patient context can trigger a rule set when a decision node is reached, and fire the relevant rules in that set. Fired rules will generate new facts that lead to further rule firings from a chaining of rules. A provider action can also be considered as a health care service that involves a provider, and includes logistic, resource, financial and time perspectives. For example, blood test and heart surgery are both provider activities that might be offered by different providers. For such care services, a patient may wish to learn more details as they relate to the four perspectives. For instance, they may like to know the location where the blood test can be performed, any necessary preparation for the test (logistics), who will perform the test (resource), what is the cost of the test and what part of it is covered by insurance (financial), and how long it takes to do the test and recover from it (time). The result of the test (e.g., blood pressure, blood lipid level), and other activities with results, are updated 
in the patient's PHR for use as additional context. Next, we describe the components of this framework.

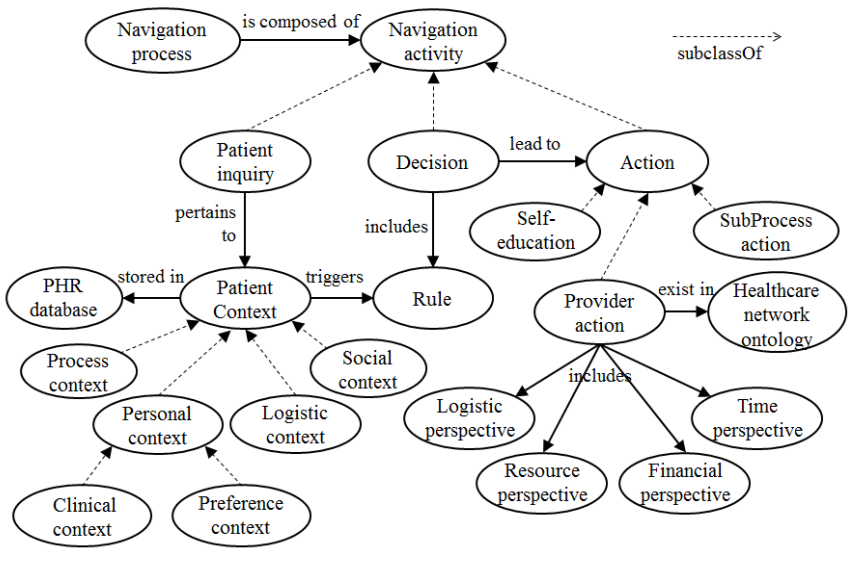

Figure 2. A conceptual map for the CANE framework

\section{A. Patient Context Model}

According to Dey [20], context is any information that is used to characterize the situation of an entity. Moreover, a system is context-aware if it uses context to bring to bear in the provision of relevant information and/or services, where relevance is naturally situation dependent. In this study, we define context as any information that characterizes the patient and is used for patient navigation and their health decisions (e.g., use of health care resources, services, and interventions).

Table 1 summarizes five categories of context and their impact on patient navigation:

- Personal context (clinical) concerns the consumer's clinical information, including personal health records, medical and long-term care plans, medical needs, mobility status, etc.

- Personal context (preferences) refers to the consumer's non-clinical information such as consumer preferences and values. This may reflect their treatment preferences (aggressive vs. moderate), cultural beliefs (e.g. veganism), interests (e.g. yoga, running), goals (e.g. want active lifestyle), etc. These preferences will influence their choices of care plans, providers and treatment methods.

- $\quad$ Process context deals with the current health and wellness issues, which are managed in the running processes that the consumer is participating in and the data (e.g., resources) associated with them. Process context allows us to anticipate the next step and show relevant services.

- Logistic context refers to any information related to the patient logistics, such as her current physical location in a hospital, and the available transportation options to travel home or elsewhere. It enables the delivery of logistic services such as GPS directions, etc.

- Social context concerns an individual's family and friends, who may be acting as a human navigator on behalf of the patient. A patient may give them permission to access her records and take some or all decisions on her behalf.
In many cases, we need to associate context from different categories for patient navigation and education. For example, guiding a patient through the discharge process requires a confluence of patient preferences, current health issues, available transportation options, and the discharge guidelines of a particular care facility.

TABLE I. PATIENT CONTEXT AND ITS IMPACT ON NAVIGATION

\begin{tabular}{|c|c|c|c|}
\hline Category & Context & Example & $\begin{array}{l}\text { Impact on patient navigation and } \\
\text { decision making }\end{array}$ \\
\hline \multirow{4}{*}{$\begin{array}{l}\text { Personal } \\
\text { context } \\
\text { (clinical) }\end{array}$} & $\begin{array}{l}\text { Personal health } \\
\text { records }\end{array}$ & $\begin{array}{l}\text { Penicillin } \\
\text { allergy }\end{array}$ & $\begin{array}{l}\text { Mark an alert in alternatives that involve } \\
\text { penicillin medication }\end{array}$ \\
\hline & $\begin{array}{l}\text { Health \& } \\
\text { wellness issues }\end{array}$ & Diabetes & Control daily sugar intake \\
\hline & Mobility status & Need crutches & Show facilities with wheelchair services \\
\hline & Habits & Smoking & Recommend quit-smoking programs \\
\hline \multirow{4}{*}{$\begin{array}{l}\text { Personal } \\
\text { context } \\
\text { (prefe- } \\
\text { rences) }\end{array}$} & Treatment style & Less aggressive & Use medication instead of surgery \\
\hline & $\begin{array}{l}\text { Medication } \\
\text { type }\end{array}$ & $\begin{array}{l}\text { Liquid > capsule } \\
>\text { caplet }\end{array}$ & Suggest liquid medicine as possible \\
\hline & Diet preference & Vegan diet & $\begin{array}{l}\text { Find nutritious sources of vegan food } \\
\text { and recommend vegan menu }\end{array}$ \\
\hline & $\begin{array}{l}\text { Activity } \\
\text { interests }\end{array}$ & Yoga, running & $\begin{array}{l}\text { Recommend a yoga or jogging facility } \\
\text { nearby }\end{array}$ \\
\hline \multirow{2}{*}{$\begin{array}{l}\text { Process } \\
\text { context }\end{array}$} & Current step & Waiting & Show educational materials \\
\hline & Next step & Examination & Show direction to the doctor's office \\
\hline \multirow{3}{*}{$\begin{array}{l}\text { Logistic } \\
\text { context }\end{array}$} & Location & Waiting room & Show entertainment services nearby \\
\hline & Time & $12: 00 \mathrm{PM}$ & Show lunch services \\
\hline & $\begin{array}{l}\text { Transportation } \\
\text { options }\end{array}$ & Shuttle and taxi & Show transportation services \\
\hline $\begin{array}{l}\text { Social } \\
\text { context }\end{array}$ & $\begin{array}{l}\text { Family and } \\
\text { friends }\end{array}$ & $\begin{array}{l}\text { Husband in a } \\
\text { heart surgery }\end{array}$ & $\begin{array}{l}\text { Show his current status and what post- } \\
\text { operative care he will need }\end{array}$ \\
\hline
\end{tabular}

\section{B. A Meta-model for Navigation Activities}

Figure 3 gives a meta model for various types of navigation activities. The meta model presents a systems oriented view detailing interactions with various system entities.

A navigation process is composed of two types of navigation activities, a decision and an action. A decision activity presents multiple options, and it includes a human decision (e.g., an explicit choice made by a patient to manage possible complications) and a rule decision (e.g., an automatic system decision by applying a rule such as directing the pregnant patient to another guideline). Based on the decision made, the system may carry out an action, e.g., GetDataAction, SubProcessAction, and MessageAction. A PatientInquiry can collect various contexts, such as body weight, eating habits and preferences, while a PHRQuery can retrieve patient data directly from the PHR system. A SubProcessAction triggers another relevant navigation process that may involve additional guidelines. For example, a patient diagnosed with type 2 diabetes may also have hypertension. A MessageAction delivers recommendations and suggestions to the patient based on her context, including EducationAction for addressing the health literacy issue, and ProviderAction for activities that involve the provider.

In this study, we focus on the decision activities and two action activities: PatientInquiry and ProviderAction. Decision activities allow us to select the subsequent pathways based on patient context. The PatientInquiry action allows the system to build the patient context by conversing with the patient. Next, the context is used for generating patient-specific actions. A ProviderAction generates a message that urges patients to interact with providers (e.g., to visit a doctor and enquire about getting a blood test). It can support patient navigation through the healthcare system consisting of many providers. Various 
provider actions along with their different perspectives will be described in the next section.

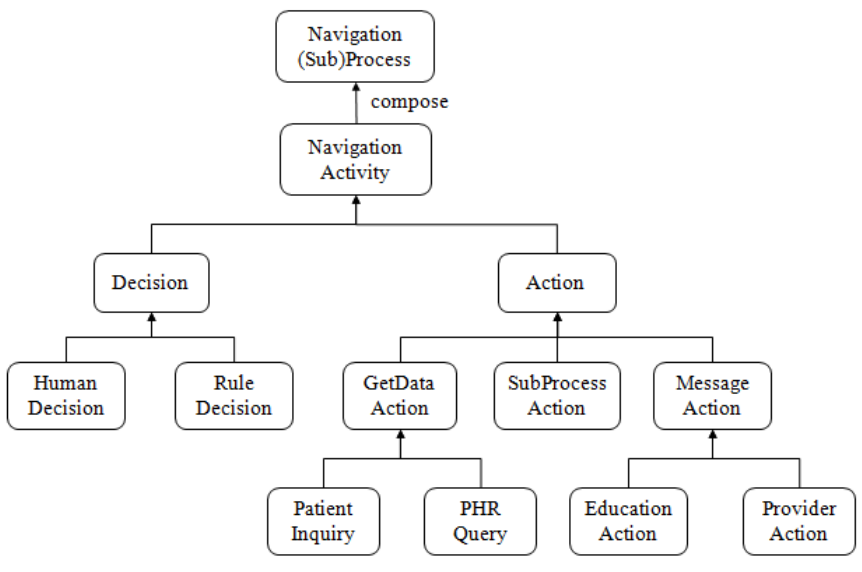

Figure 3. A meta model for navigation activities

\section{Healthcare Network Ontology}

In this study, we propose a healthcare network ontology model to identify the roles of people, places, and things - from multiple perspectives (e.g., resource, logistics, financial, time, etc.), involved in actions as discussed above. It assists patients when navigating provider actions (as shown in Figure 3). Ontologies are explicit formal specifications of the terms in a domain and the relationships among them. They facilitate knowledge sharing, logical inference, and knowledge reuse. The Web Ontology Language (OWL) [21] is a semantic markup language for publishing and sharing ontologies on the Web. Thus, OWL-encoded ontologies are sharable across various departments and organizations. Since OWL uses firstorder logic, the class models and descriptions of data attributes, constraints and relationships in these models can be formally verified. It can be used to detect inconsistencies in the model and infer new information by correlating this data, and thus lends itself to machine reasoning. We encode the healthcare network model in OWL using Protégé 3.4 [22], a popular tool for ontology editing and representation. This model is shared across the healthcare enterprise.

Figure 4 shows a partial representation of our healthcare network ontology model. It describes the main entities of interest, their properties, and the semantic relationships among them. It captures various provider activities in a hierarchy and their relationships with resources, healthcare providers, costs, etc. Their relationships are described so as to capture the constraints between these entities, such as relation "providedBy" between ProviderActivity and HealthcareProvider. ProviderActivity includes two subclasses MedicalTask and AdministrativeTask, both of which have a detailed hierarchy. A health care provider can be an institution such as a "HealthFacility" or an individual "HealthWorker". The class HealthFacility has subclasses including Pharmacy, MedicalNursingHome, HealthCareCenter, Hospital, and MedicalLab. These subclasses can be further categorized into more refined health institutions. The concept of health worker is categorized in the same way, depending upon the type of medical care the worker provides.
Our healthcare network model is represented by OWL-DL, which is a subset of OWL with reasonable inference capability. It follows an object-oriented approach to describe the structure of a domain in terms of classes, their properties and semantic relationships. In Figure 4, each concept is a class that defines an important entity in this domain. For example, we define classes HealthCareProvider, and MedicalTask as follows:

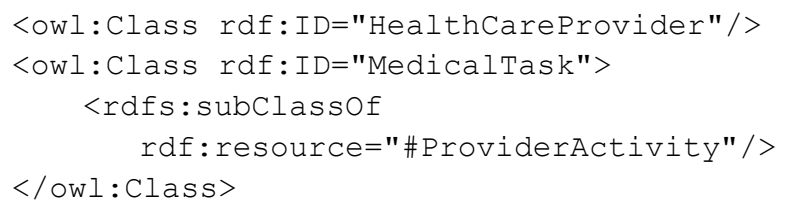

Note that the class MedicalTask is defined as a subclass of ProviderActivity by using rdfs: subclassof. Class is associated with its attributes through Datatype property. One such property of the class ProviderActivity is hascost, represented as follows:

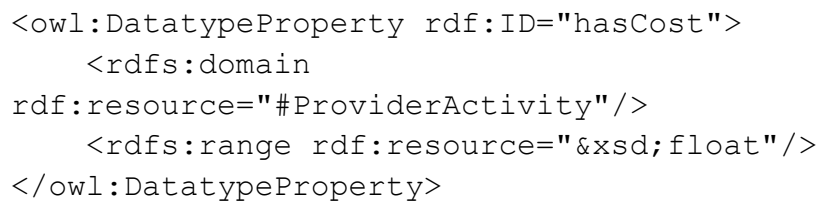

A class can also link with other classes through Object property. For example, the class ProviderActivity is associated with the class HealthCareProvider by property providedBy.

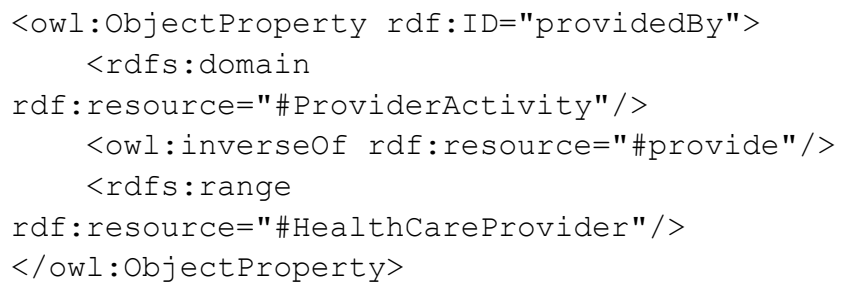

Finally, an individual represents an instance of a class. The example below shows an X-ray test provided by Hospital_A using machine $X-r a y \_m a c h i n e \_003$ costing $\$ 85$.

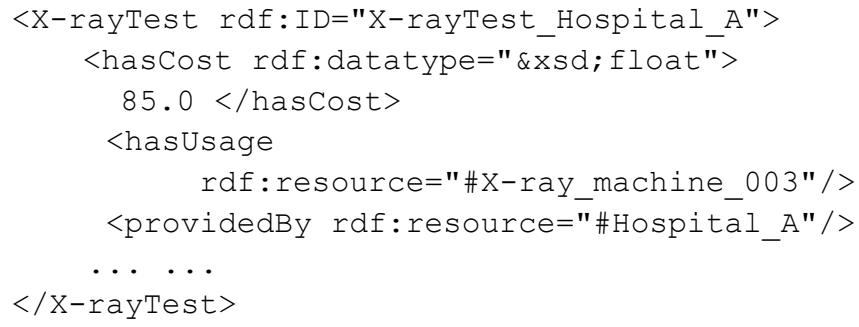

By using SPARQL queries [23], we can retrieve and manipulate data stored in the ontology database. For example, SPARQL queries can model patient questions such as "What treatments are available for type 2 diabetes?" and "List all the healthcare providers that offer chest X-ray tests and compare their costs." This makes it possible to extract relevant information pertaining to the current patient context. 


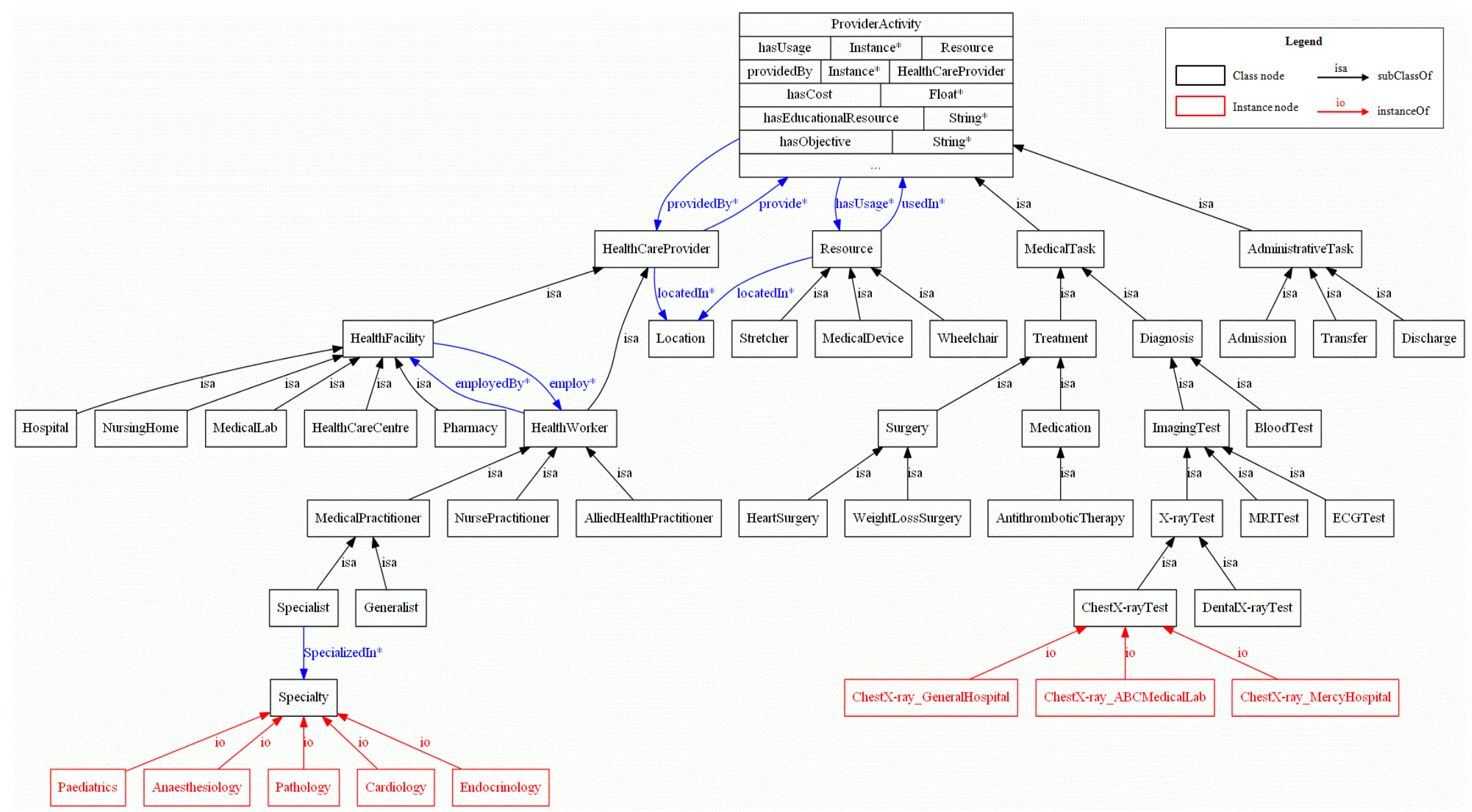

Figure 4. Partial representation of the healthcare network ontology 
Finally, domain ontologies from medical disciplines are included in our knowledge framework since they are critical for decision support. Our application of a healthcare network ontology in support of navigation is analogous to the application of medical ontologies such as SNOMED CT [24] for defining clinical terms. It enables a common understanding of terms across the healthcare enterprise.

\section{CONTEXT-AWARE NAVIGATION}

By capturing the navigation process in a workflow model, and provider-related medical services in the form of a health network ontology, we enable context-aware patient navigation, based on their medical situation (e.g., deciding which guideline to follow, whether to see a doctor, etc.), and their preferences (e.g., deciding to focus on exercise or diet). In addition to selfmanagement regarding clinical issues, we allow patients to learn about many different perspectives captured in the health network ontology of the health care services offered by a variety of providers.

\section{A. Navigation in the Healthcare Network}

Using the healthcare network ontology shown in Figure 4, patients are able to navigate various perspectives related to health care services, such as their care providers, location(s), resources, costs, etc. For example, in the subprocess SP4 (assess and manage cardiovascular risks) or SP5 (assess and manage long term complications) from Figure 1, a patient may be advised to take a chest X-ray test if heart disease or certain complications are detected. Our ontology in Figure 4 facilitates a patient's understanding of such care services. As described above, SPARQL queries [23] allow us to retrieve the ontology information pertaining to the current patient context during the navigation. Thus, the information regarding chest X-ray tests will only be retrieved and displayed whenever needed (i.e., SPARQL queries are only triggered when patients are suspected of heart disease or certain complications). Similarly, we can retrieve only the cost data for comparing the costs of different chest X-ray tests offered by various providers. Thus, this ontology directs patient navigation in a specific, context-aware manner, and makes it more efficient.

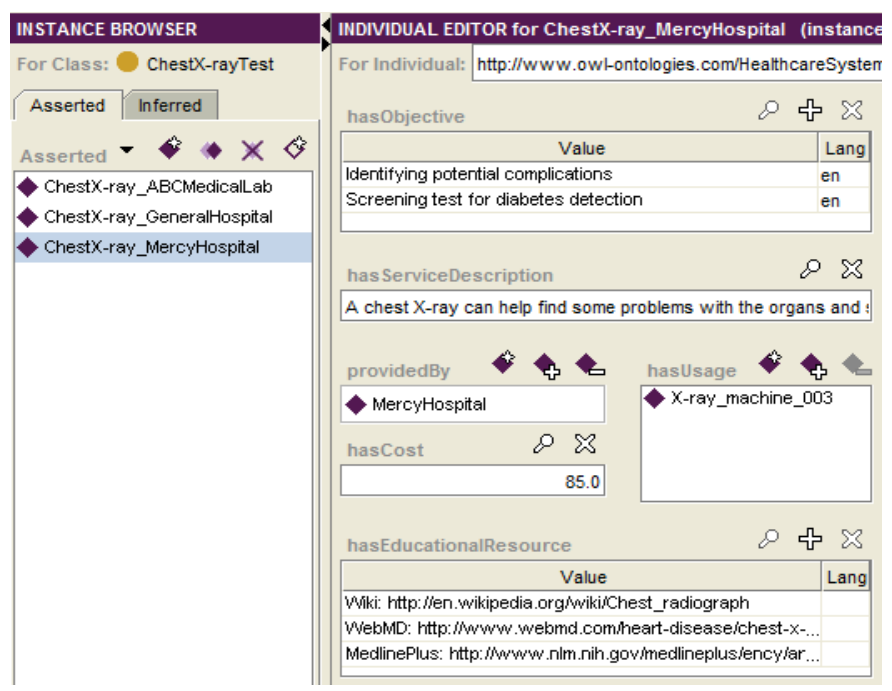

Figure 5. A Protégé screenshot of a provider activity
Figure 5 shows example provider activities, which are stored and maintained in our ontological database, in Protégé 3.4 [22]. The left panel shows three service providers for chest $\mathrm{X}$-ray tests. The details about the chest X-ray test offered by MercyHospital are shown in the right panel. The patient can learn about the objective of a chest X-ray test in the context of diabetes management, the description of this service, the provider and the provider location(s), customer satisfaction and other ratings related to this provider, the cost, and the medical devices and other resources used in this service. Further, educational resources include links from health authorities such as WebMD and MedlinePlus, and common knowledge bases like Wikipedia.

\section{B. Rules for Context-aware Decision Making}

Context-aware routing of navigation activities is enabled by two types of nodes: rule task for deriving new patient context using rule-based reasoning, and control node for deciding which path to take based on the patient context.

A rule task contains one or more rules that embody medical knowledge and is used to make complex decisions in navigation processes through logical reasoning. A rule can be written as rule_id: If conditions $\rightarrow$ Then actions. Once the conditions are met, the corresponding rule is fired and it produces actions. Conditions can be connected by the $A N D$, $O R$, and NOT logical operators to define complex conditions. Of course, more parameters can also be used such as rule group for clustering rules, and priority for resolving conflicts. The node T5 in Figure 1 is an example rule task for evaluating medical conditions of the patient. It may contain the following rules R1-R3, where R3 uses the results from R2 using rule-based forward chaining. Note the term ACR in rule R2 and R3 stands for Albumin Creatinine Ratio (also known as microalbuminuria). Thus, rules will generate high-level patient context that is used in determining the appropriate navigation paths that should be followed.

\section{R1: If (patient.hasHeartAttackHistory = "yes") OR (patient.smoking = "yes") OR (patient.bloodPressure $>145 / 90 \mathrm{mmHg}$ ) OR ((patient.physicallnactivity = "yes") AND (patient.age > 50)) $\rightarrow$ Then patient.addPotentialHealthlssue ("Heart disease")}

R2: If ((patient.ACR > $2.5 \mathrm{mg} / \mathrm{mmol})$ AND (patient.gender = "Male")) OR ((patient.ACR > $3.5 \mathrm{mg} / \mathrm{mmol})$ AND (patient.gender = "Female")) $\rightarrow$ Then patient.hasAbnormalACR = "yes"

\section{R3: If (patient.hasAbnormalACR = "yes") OR (patient.hasHematuria = "yes") OR (patient.hasAbnormal_AlbuminExcretionRate = "yes") \\ $\rightarrow$ Then patient.addPotentialHealthlssue ("Kidney complication")}

A navigation process may include scenarios that address the needs of many different patients. However, only certain activities will be triggered for each specific patient. This is realized by the control node which selects subsequent navigation activities according to specific patient context. The running example in Figure 1 includes five decision nodes: N1$\mathrm{N} 3$ and N5-N6. For instance, N1 is a decision node to decide the next step (trigger another navigation process for a pregnant diabetes patient or use the current process for a regular patient) based on her pregnancy status. Similarly, N2 relies upon the patient's logistics context, while N3 makes a decision based on 
the patient's clinical context (i.e., whether she has high blood glucose, heart-related issues, or potential complications) and preference context (i.e., whether she is interested in receiving education on maintaining a healthy lifestyle). The context used in these control nodes is usually obtained from the PHR database or the high-level context produced by rule tasks. Thus, integrating these rules and applying results from rule-based reasoning into a navigation process along with the control nodes is critical for guiding patients according to evidencebased best practice.

\section{An Example Navigation Scenario}

Figure 6 shows how a personalized navigation path evolves for a specific patient. The dotted line shows patient interactions with the system using the model in Figure 1 (some lines are omitted due to space limitations). The patient answers questions that are used to assess their current situation. First, various clinical context information are collected through patient conversation (e.g., T1, T2, and T4). Here the evaluation results (T5) show that the patient has no heart-related problems or high blood glucose. T5 is a rule task associated with a rule set (e.g., rule R1-R3 in the above section) that embodies relevant medical knowledge. In this scenario, $\mathrm{R} 1$ and $\mathrm{R} 2$ are not fired since the conditions are not met, while R3 is fired and accordingly triggers subprocess SP11 for managing chronic kidney damage.

Patients can also express their preferences and value. Since the patient has concerns about potential complications and would like to create a healthy lifestyle, two educational activities are presented that are followed by more detailed management subprocesses. Then patient preference data is collected to provide her with customized self-management services (i.e., "jogging program" and "healthy menu program"). Such services can be linked to smart phone apps as well. Regarding long term complications, Subprocess SP5 is a BPMN 2.0 adhoc subprocess that can invoke one or more nested subprocesses SP11-SP15 in any order as per the context and needs of the patient. Subprocesses may relate to additional guidelines. For example, SP11 requires kidney related medical knowledge, while SP12 refers to guidelines for eye related diseases.

\section{IMPLEMENTATION PLAN}

The CANE system is a part of our larger project "streamlined patient experience using a formal process-driven approach" [11], which considers both patient navigation and patient-provider communication in the care process. In this study, we focused on the design and development of a patient engagement platform to help patients navigate through their care processes by application of formal medical guideline knowledge, and driven by their context.

Figure 7 depicts an implementation architecture for the CANE framework based on our conceptual model in Figure 2. Several roles are involved in this system. Process designers use a workflow modeling tool to create various navigation processes for different medical issues (e.g., diabetes, heart disease, chronic cough) to be stored in the process repository. They are initialized and executed by the workflow engine to support the patient navigation process. Meanwhile, the knowledge engineers collect information about care services offered by various providers. They may need to communicate with health professionals and consult other medical resources as well. Then they use an ontology editor (e.g., Protégé 3.4) to build and maintain the health care network ontology that captures provider activities (i.e., care services), health providers, resources, etc., and the relationships among them.

At runtime, a navigation process instance is executed and fed with specific patient context. The jBPM workflow engine [25] is responsible for maintaining the status of all running process instances and coordinating with other components. jBPM also integrates with a rule engine, which is responsible for rule-based reasoning for the rule task. The rule engine triggers rules, evaluates conditions, and asserts new facts that can trigger other actions accordingly. The workflow engine interfaces with the patient through the navigation GUI and collects new/updated patient context through patient inquiries. We augmented the PHR database with patient preference data, other types of context, and the navigation logs recorded by the workflow engine. The workflow engine can interact with the context manager to retrieve/update patient context (e.g., medical history) from/in the PHR database. The workflow engine can also query knowledge about provider activities regarding their resources, costs, etc. from the healthcare network ontology through the ontology manager. Thus, various navigation activities, such as self-care, provider-care services, etc. occur in a context-aware manner through a webbased management console and as the patients interact with it.

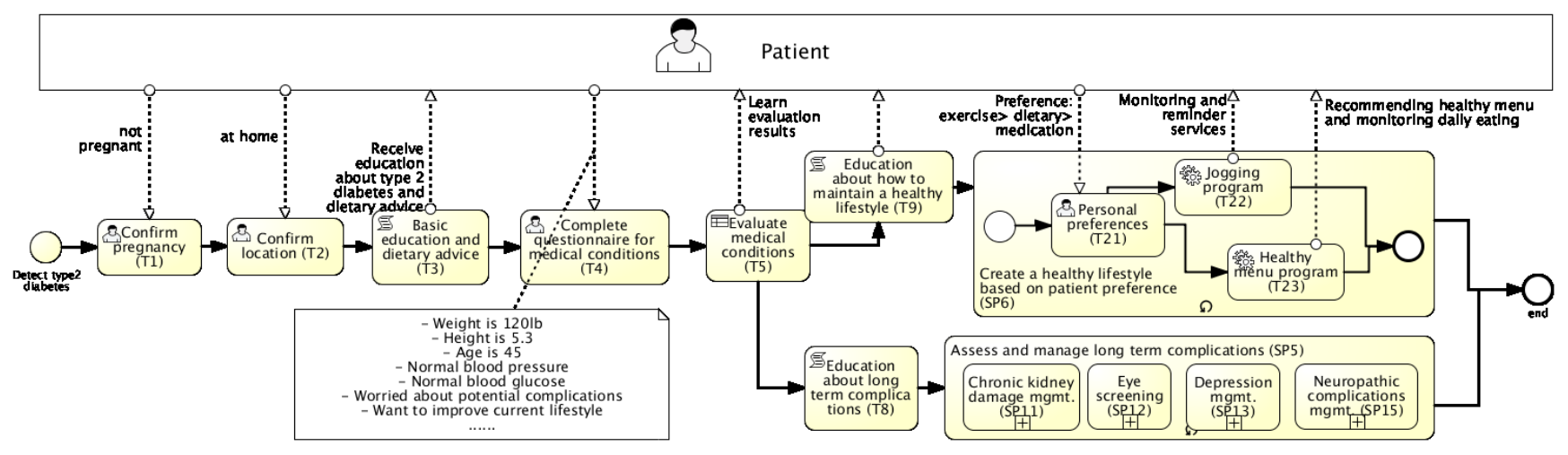

Figure 6. A patient navigation instance 


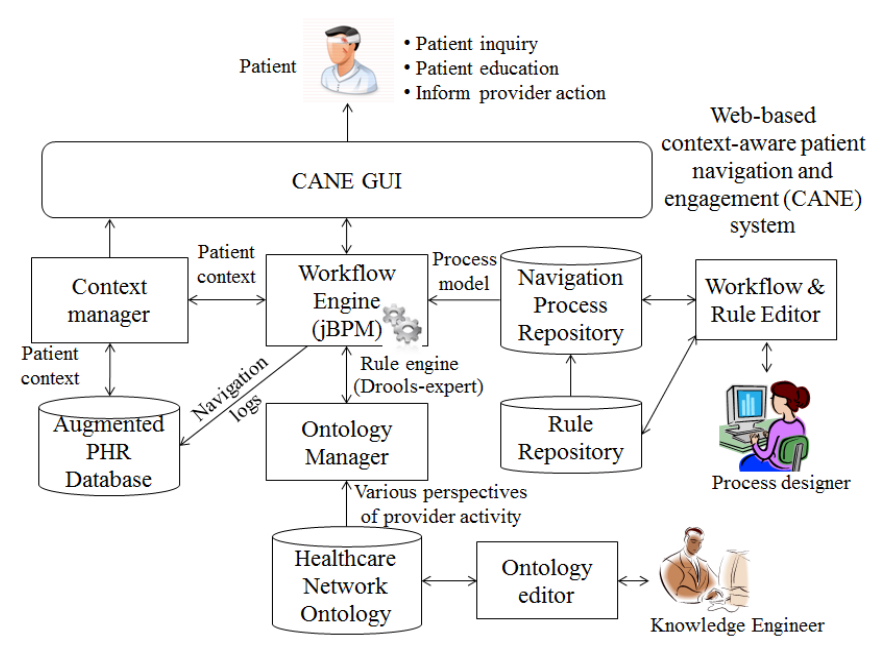

Figure 7. A system architecture for CANE

Navigation activities and their results are logged in the patient's PHR and are labeled according to guidelines, clinical ontologies, and the healthcare network ontology. The activities may use clinical terms from ontologies such as SNOMED CT and may involve interactions with various providers in the health care enterprise. The providers have identities and are categorized using the healthcare network ontology.

We are currently building this patient engagement platform by leveraging the open source PHR tool MyOSCAR. We plan to test the usability of this system for type 2 diabetes patients by conducting an empirical study that evaluates the impact of CANE on patient experience. The next step is to allow this patient-centric application to use the services provided by Fusion [26], which is a cloud-based platform for large-scale, low-cost management of healthcare data. Fusion enables secure storing and sharing of EHR records among healthcare providers, patients, payers, and researchers, and a prototype of Fusion is currently being built on HP Cloud Compute. We will leverage this infrastructure to enhance the CANE application. With patient consent, de-identified data gathered over a large number of patients through Fusion can be used to support patient navigation, and improve patient flow, patient satisfaction, and health service coordination and integration. These traces can be used to study the actual processes of patients in a healthcare enterprise. Further, these data logs can also be used to support decision making for a patient by providing aggregate, visual data from their cohort (i.e., patients with a similar profile). For example, we can present a breakdown of the types of treatment methods chosen by patients with similar conditions by percentage and their corresponding effectiveness or patient satisfaction rate.

\section{RELATED WORK}

In this section, we organize the related work into the following three categories.

Clinical Guideline Modeling. Medical guidelines were originally in the form of free-format text documents to assist medical decision making during diagnosis, management and treatment within different areas of healthcare. Computer-
Interpretable Guidelines (CIGs) can produce personalized recommendation during patient encounters and reduce variance in patient treatment. Recently, various approaches have been proposed to represent CIGs, such as Arden Syntax, Asbru, EON, GLIF, GUIDE, PROforma, and SAGE, reviewed in $[8,9]$. These approaches represent clinical guidelines as plans, whose components include decisions, actions, and their relationships. Decision steps are used for conditional and unconditional routing of the flow, while action steps are used to specify a set of tasks or a sub-plan to be carried out. Peleg et al. [8] reviewed six CIG modeling techniques and established a consensus on a set of common components. For a more comprehensive review of systems using CIGs, see [9].

Since CIGs were originally designed for clinical decision making (i.e., for health professionals rather than patients), they are unsuitable for our purpose. In this study, we use BPMN 2.0 for modeling and executing navigation processes because in addition to the medical activity types, it also provides service tasks, human tasks, script tasks, etc. for modeling navigation activities.

Patient Care Information Systems. Recent years have seen an increasing interest in Patient Care Information Systems (PCISs), such as PHR systems, which allow patients to store, view, and share their medical histories, medications, lab results, etc. to better manage their health information and communicate with their care providers [6,7]. A selection of web-based PHR applications such as WebMD is reviewed in [6]. Other PCISs are devoted towards patient participation and decision making. For example, an asthma kiosk application was designed to capture critical information to drive guidelinebased care for pediatric asthma [27]. HealthWeaver Mobile smart phones manage information related to a specific health issue such as cancer [28]. Other systems or applications have been developed to manage general wellness, e.g., a fitness program tailored to personal preferences is discussed in [29].

However, engaging consumers in the health care system is more than just managing and communicating their health records. Patients should learn relevant medical knowledge about the healthcare system pertaining to their context, and be able to understand and interpret their own health issues. Some tools or websites provide such help: e.g. Patient Decision Aids [30] for providing health resources for treatment options, recovery plans, etc. and U.S. Health \& Human Services [31] for comparing care providers in terms of their distance, hospital type, emergency services, etc. However, they are generic and not context-aware.

Human Navigators. The role of a human navigator for a patient was researched by many studies $[5,32,33]$ as a way to reduce health disparities and improve treatment for chronic diseases. For example, [5] shows a navigator can improve cancer outcome by providing screening to patients regardless of ability to pay, guiding them to a timely diagnosis and treatment, and increasing outreach and public education. A literature review [32] showed that there is evidence supporting the role of human navigators to improve many aspects of breast cancer care. On the other hand, [33] raised doubts about the efficacy and cost-effectiveness of human navigators in improving cancer care. 
Although most related work on human navigators concerns cancer care since it is a chronic and costly disease, navigation plays a critical role in other medical disciplines such as diabetes for improving the care quality and health literacy [14]. Promoting automated navigation for chronic illnesses will not only improve patients' health condition, but also reduce the overall healthcare cost. Thus, there is a need to develop an information technology-based patient navigation system that can cover a variety of chronic illnesses and general issues, and guide consumers through their care processes. We expect it to complement human navigators by offering many of the advantages to a much broader community at a fraction of the cost and reduce the burden on human navigators.

\section{SUMMARY AND CONCLUSIONS}

In this paper, we proposed a context-aware patient navigation and engagement framework to assist patients in their decision making and self-management. We use knowledge from several research areas including: (1) the clinical domain, for understanding the medical knowledge and guidelines; (2) the public health domain, for understanding the providers, services and their networking in the healthcare system; (3) consumer health informatics on health literacy and consumer education; and (4) medical informatics, to provide the technologies for capturing these contexts and driving context-aware patient navigation.

We aim to help patients, especially those with chronic illnesses, to navigate the medical guidelines and interact with the complex health care system that involves various care providers, and their resources and services. In this approach, patient-centered care is realized by constructing various patient contexts from their health record, personal preferences, lifestyles, social contacts, etc., and using them to traverse relevant medical guidelines step-by-step. We are currently implementing this patient engagement platform and aim to do an empirical evaluation as part of future work.

Finally, we observe that a system such as CANE would benefit from integration of the healthcare provider ontology proposed in Figure 4 with a standard medical ontology of clinical terms such as SNOMED CT [24]. Medical guidelines would benefit considerably from these combined ontologies. It would enhance interoperability and consistency, assist in the better definition of navigation processes, and further facilitate the development of rules to support patient navigation.

\section{ACKNOWLEDGEMENT}

We thank Dr. Wei-Nchih Lee for validating the clinical use case and his helpful comments and suggestions on this paper.

\section{REFERENCES}

[1] Purdue University, "A Healthcare-Delivery System for the Next Generation," 2006.

[2] T. Johnson. (2010). Healthcare Costs and U.S. Competitiveness. Available: http://www.cfr.org/health-science-and-technology/healthcare-costsus-competitiveness/p13325.

[3] J. H. Hibbard, "Engaging health care consumers to improve the quality of care," Medical Care, vol. 41, pp. I61-70, 2003.

[4] S. Sofaer, "Navigating Poorly Charted Territory : Patient Dilemmas in Health Care "Nonsystems"," Medical Care Research and Review, vol. 66, pp. 75s-93s, 2008.
[5] H. P. Freeman, "Patient Navigation: A Community Based Strategy to Reduce Cancer Disparities," J Urban Health, vol. 83, pp. 139-141, 2006.

[6] M. I. Kim and K. B. Johnson, "Personal Health Records: Evaluation of Functionality and Utility," J Am Med Inform Assoc, vol. 9, pp. 171-180, 2002.

[7] P. C. Tang, et al., "Personal Health Records: Definitions, Benefits, and Strategies for Overcoming Barriers to Adoption," J Am Med Inform Assoc vol. 13, pp. 121-126, 2006.

[8] M. Peleg, et al., "Comparing computer-interpretable guideline models: A case-study approach," Journal of the American Medical Informatics Association, vol. 10, pp. 52-68, 2003.

[9] D. Isern and A. Moreno, "Computer-based execution of clinical guidelines: A review," International Journal of Medical Informatics, vol. 77, pp. 787-808, 2008.

[10] National Institute for Health and Clinical Excellence. NICE Pathways. Available: http://pathways.nice.org.uk/.

[11] W. Yao, et al., "Towards a Formal Process-driven Framework for Streamlining Patient-centric Care," in Proceedings of CAiSE'12 Forum, Gdańsk, Poland, 2012, pp. 179-186.

[12] OMG, "Business Process Model And Notation (BPMN) Version 2.0", January 2011. Available: http://www.omg.org/spec/BPMN/2.0/PDF.

[13] J. H. Robinson, et al., "Patient-centered care and adherence: Definitions and applications to improve outcomes," Journal of the American Academy of Nurse Practitioners, vol. 20, pp. 600-607, 2008

[14] A. T. McCray, "Application of Information Technology: Promoting Health Literacy," J Am Med Inform Assoc vol. 12, pp. 152-163, 2005.

[15] A. Fux, et al., "How can personal context improve adherence to clinical guidelines?," in Proceedings of ProHealth'12 / KR4HC'12, Tallinn, Estonia, 2012, pp. 14-19.

[16] J. Hong, et al., "Context-aware systems: A literature review and classification," Expert Systems with Applications, vol. 36, pp. 8509-8522, 2009.

[17] M. Baldauf, et al., "A survey on context-aware systems," Int. J. Ad Hoc and Ubiquitous Computing, vol. 2, 2007.

[18] J. E. Bardram, "Applications of Context-Aware Computing in Hospital Work - Examples and Design Principles," in 2004 ACM Symposium on Applied Computing, Nicosia, Cyprus, 2004.

[19] T. H. Maugh. (2007). Counting the cost of Type 2 diabetes. Available: http://articles.latimes.com/2007/apr/11/science/sci-diabetes11

[20] A. K. Dey, "Understanding and using context," Personal Ubiquitous Comput., vol. 5, pp. 4-7, 2001.

[21] S. Bechhofer, et al., "OWL Web Ontology Language Reference," presented at the W3C Recommendation, 2004.

[22] Stanford University. (2009). Protégé: An open source ontology editor and knowledge-based framework (3.4). Available: http://protege.stanford.edu/.

[23] E. Prud'hommeaux and A. Seaborne, "SPARQL Query Language for RDF," W3C working draft, vol. 4, 2008.

[24] International Health Terminology Starndard Developmen Organization. SNOMED Clinical Terms. Available: http://www.ihtsdo.org/snomed-ct/.

[25] JBoss Community. jBPM5. Available: http://www.jboss.org/jbpm.

[26] S. Basu, et al., "Fusion: Managing Healthcare Records at Cloud Scale," IEEE Computer, Special Issue on Move Toward Electronic Health Records, forthcoming, 2012.

[27] S. C. Porter, et al., "The asthma kiosk: a patient-centered technology for collaborative decision support in the emergency department," Journal of the American Medical Informatics Association, vol. 11, pp. 458-467, 2004.

[28] P. Klasnja, et al., "Supporting cancer patients' unanchored health information management with mobile technology," in the AMIA Annual Symposium Proceedings, 2011.

[29] A. Chapko, et al., "uService - Enabling user-driven fitness services onthe-go," in Proceedings of AMCIS'11, 2011.

[30] The Patient Decision Aids Research Group. Patient Decision Aids. Available: http://decisionaid.ohri.ca/.

[31] U.S. Department of Health \& Human Services. Hospital Compare. Available: http:/hospitalcompare.hhs.gov/.

[32] S. Robinson-White, et al., "Patient Navigation in Breast Cancer," Cancer Nursing, vol. 33, pp. 127-140, 2010.

[33] K. J. Wells, et al., "Patient navigation: state of the art, or is it science?," Cancer, vol. 113, pp. 1999-2010, 2008. 